\title{
Inclusive and differential single-top cross sections from ATLAS and CMS
}

\section{Barbara Alvarez Gonzalez ${ }^{a}$ on behalf of the ATLAS and CMS Collaborations}

aInstituto Universitario de Ciencias y Tecnologías Espaciales de Asturias (ICTEA), Universidad de Oviedo, Oviedo, Spain

E-mail: balvarez@ern.ch

\begin{abstract}
An overview of the most recent single-top-quark cross section results carried out by the ATLAS and CMS experiments of the CERN LHC is presented in this document. The latest $t$-channel and tW production cross section results at $13 \mathrm{TeV}$ are shown together with the ATLAS and CMS single-top-quark cross section combinations at 7 and $8 \mathrm{TeV}$. The observation of the associated production of a top quark and a $\mathrm{Z}$ boson at $13 \mathrm{TeV}$ is also presented. All measurements are consistent with the SM predictions. These results demonstrate a good understanding of the electroweak production mechanism of single-top-quarks providing important constraints on interference models and better understanding of the structure of the proton. More data and higher production cross sections at the LHC allow us to probe differentially the single-top-quark processes; some differential cross section measurements are already performed and discussed here as well.
\end{abstract}




\section{Introduction}

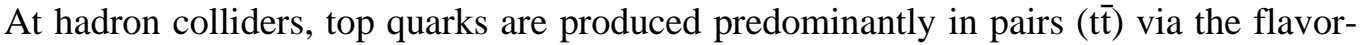
conserving strong interaction. Alternatively, top quarks can be also produced singly through the electroweak interaction via three production modes: $t$-channel, $s$-channel and $\mathrm{tW}$. The study of single-top-quark production is interesting because of its sensitivity to the $\mathrm{V}_{\mathrm{tb}}$ coupling and as a probe for anomalous Wtb coupling and flavor changing neutral current in production.

In this document an overview of the most recent single-top-quark cross section results is presented. Note that all measurements are performed in proton-proton collisions at a centre-of-mass energy of $13 \mathrm{TeV}$ by the ATLAS [1] and the CMS [2] experiments. The combinations of singletop-quark production cross section measurements are done at centre-of-mass energies of 7 and 8 $\mathrm{TeV}$.

\section{Combinations of production cross-section measurements and $\left|f_{\mathrm{LV}} V_{t b}\right|$ determinations}

The combinations of single-top-quark production cross section measurements by ATLAS and CMS represent the most precise measurements up to date [3]. These combinations are performed using the best linear unbiased estimator method for each production mode and per centreof-mass energy corresponding to integrated luminosities of 1.17 to $5.1 \mathrm{fb}^{-1}$ at $7 \mathrm{TeV}$ and 12.2 to $20.3 \mathrm{fb}^{-1}$ at $8 \mathrm{TeV}$.

The combined $t$-channel cross sections are $67.5 \pm 5.7 \mathrm{pb}$ and $87.7 \pm 5.8 \mathrm{pb}$ at 7 and $8 \mathrm{TeV}$, respectively. The combined tW cross sections are $16.3 \pm 4.1 \mathrm{pb}$ and $23.1 \pm 3.6 \mathrm{pb}$ at 7 and $8 \mathrm{TeV}$, respectively. For the $s$-channel cross section, the combination is performed only at $8 \mathrm{TeV}$ and yields $4.9 \pm 1.4 \mathrm{pb}$. The CKM matrix element $V_{t b}$ multiplied by a form factor $f_{\mathrm{LV}}$ is determined for each production mode and centre-of-mass energy, and all individual results are combined resulting in $\left|f_{\mathrm{LV}} V_{t b}\right|=1.02 \pm 0.04$ (meas) \pm 0.02 (theo). All combined measurements are consistent with their corresponding Standard Model (SM) predictions.

\section{Measurement of the tW production cross section at $13 \mathrm{TeV}$}

The study of the associated production of a single-top-quark and a $\mathrm{W}$ boson, $\mathrm{tW}$, is important due to its interference with $\mathrm{t} \overline{\mathrm{t}}$. The $\mathrm{tW}$ production cross section measurement is performed using events with one electron and one muon in the final state along with at least one jet originating from a bottom quark. The data collected by CMS used for this measurement corresponds to an integrated luminosity of $35.9 \mathrm{fb}^{-1}$ [4]. A multivariate discriminant, boosted decision tree, exploiting the kinematic properties of the events, is used to separate the signal from the dominant $t \bar{t}$ background. The measured cross section of $63.1 \pm 1.8$ (stat) \pm 6.4 (syst) \pm 2.1 (lumi) pb agrees with the SM expectation and with the results from ATLAS [5]. The dominant sources of uncertainties are related to the object reconstruction efficiencies, pileup, and the integrated luminosity.

\section{Measurement of differential tW cross sections at $13 \mathrm{TeV}$}

The tW production is also measured as normalized differential cross sections at $13 \mathrm{TeV}$ [6]. The data set collected by CMS used for this measurement is the same as the one for the tW inclusive cross section. Events containing one muon and one electron in the final state are analyzed. The presence of lower-energy jets is vetoed to reduce the background contributions. The measurements are made as a function of various properties of the event: the transverse momentum of the leading lepton in Fig.1 (left), the transverse momentum of the jet in Fig.1 (right); the difference in the $\varphi$ angle of the muon and the electron; the longitudinal momentum of the muon, the electron and the jet; the invariant mass of the muon, the electron, and the jet; and the transverse mass of the electron, the muon, the jet, and the missing transverse momentum. The resulting distributions are unfolded to particle-level and compared with calculations at next-to-leading order (NLO) in perturbative QCD. A fiducial region is defined according to the detector acceptance, and the requirement of exactly one b-tagged jet. The differential cross sections are normalized with the fiducial cross section, which cancels out several systematic uncertainties. Within current uncertainties, all the predictions agree with the data. ATLAS result can also be found in Ref. [7]. 

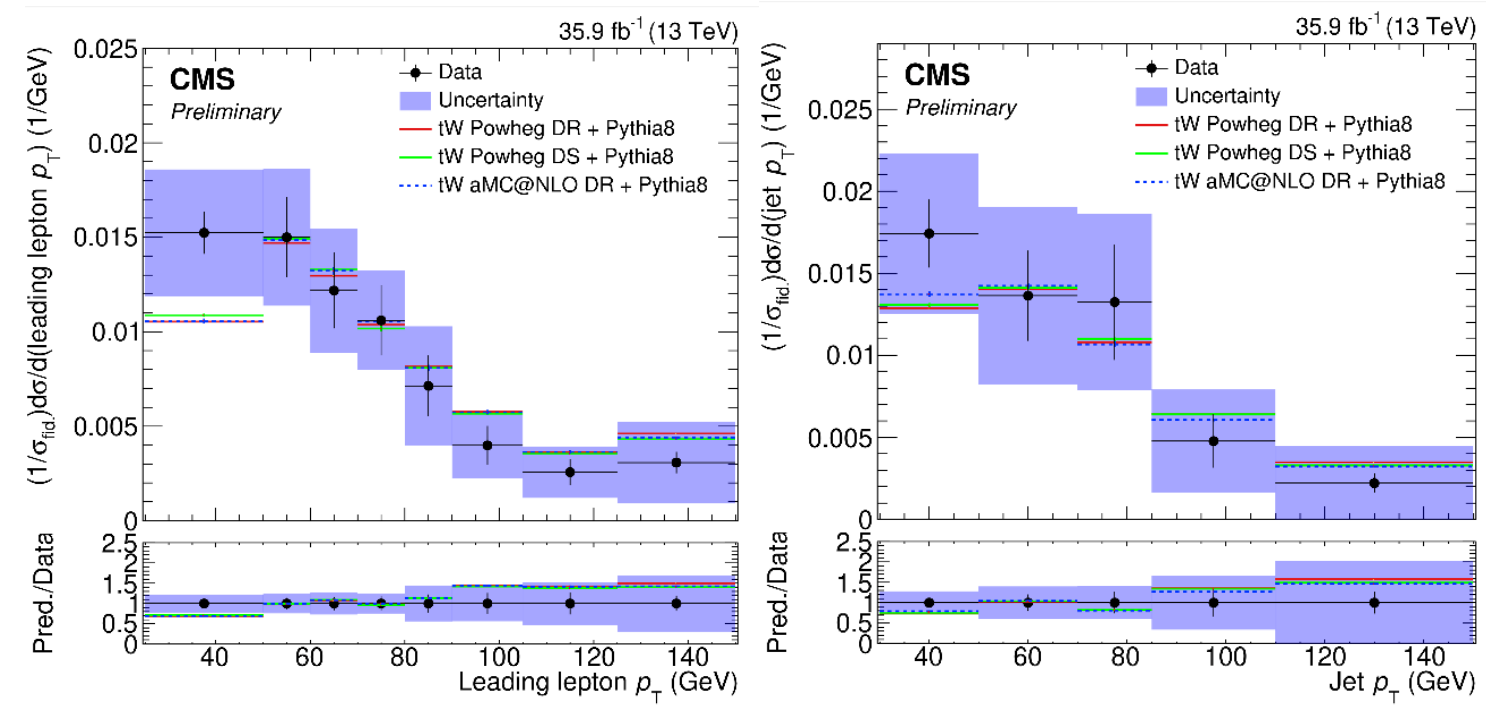

Figure 1:Normalized differential $t W$ production cross section as a function of the $p_{T}$ of the leading lepton (left) and the $p_{T}$ of the jet (right). The solid band represents the total uncertainty. Predictions from POWHEG and MadGraph5_aMC@NLO are also shown [6].

\section{Measurement of the single-top-quark and antiquark production cross sections in the $t$-chan- nel and their ratio at $13 \mathrm{TeV}$}

The cross sections for the production of single-top-quarks and antiquarks in the $t$-channel, and their ratio, are measured by CMS [8] using $35.9 \mathrm{fb}^{-1}$ of $13 \mathrm{TeV}$ data. Events with one muon or electron are selected. Different categories of jet and $b$ jet multiplicity and multivariate discriminators, boosted decision trees, are applied to separate the signal from the background. The cross sections for the $t$-channel production of single-top-quarks and antiquarks are measured to be 130 \pm 1 (stat) \pm 19 (syst) pb and $77 \pm 1$ (stat) \pm 12 (syst) pb, respectively, and their ratio, $R t$-ch, is $1.68 \pm 0.05$ (stat) \pm 0.05 (syst) pb. The results agree with the SM predictions and with the result from ATLAS that uses a smaller data set of $3 \mathrm{fb}^{-1}$ of $13 \mathrm{TeV}$ data [9].

The statistical uncertainty plays only a minor role for the achieved precision of the measurements, which are limited by the systematic uncertainties in the modeling of the signal process. Deeper understanding of these effects and improved procedures to estimate the uncertainty are therefore crucial to further decrease the systematic uncertainty. The value of $R t$-ch can be used to test the predictions from different PDF sets for their compatibility with the data.

\section{Measurement of differential cross sections and charge ratios for $t$-channel at $13 \mathrm{TeV}$}

The measurement of differential cross sections for $t$-channel single-top-quark and antiquark production by the CMS experiment is also presented [10]. From a data set corresponding to an integrated luminosity of $35.9 \mathrm{fb}^{-1}$, events containing one muon or electron and two or three jets are analyzed. The cross section is measured as a function of the top quark $p_{\mathrm{T}}$, rapidity, and polarization angle, the charged lepton $p_{\mathrm{T}}$ and rapidity, and the $p_{\mathrm{T}}$ of the $\mathrm{W}$ boson from the top quark decay.

In addition, the charge ratio is measured differentially as a function of the top quark, charged lepton, and $\mathrm{W}$ boson kinematic observables. The results agree with SM predictions using various NLO event generators and sets of parton distribution functions. Additionally, the $\mathrm{t} \overline{\mathrm{t}}$ spin asymmetry, sensitive to the top quark polarization, is determined from the differential distribution of the polarization angle at parton level to be $0.440 \pm 0.070$, in agreement with the SM prediction.

\section{Singly and doubly resonant top-quark production at $13 \mathrm{TeV}$}

The normalized differential cross sections are measured in a fiducial phase-space region where interference effects between $\mathrm{t} \overline{\mathrm{t}}$ and associated production of a single-top-quark with a $\mathrm{W}$ boson and a b-quark, tWb, are significant. Events with exactly two leptons and two b-tagged jets 
that satisfy a multiparticle invariant mass requirement are selected using $36.1 \mathrm{fb}^{-1}$ of $13 \mathrm{TeV}$ data with the ATLAS detector [11]. The results are compared with predictions from simulations using various strategies for the interference as shown in Fig. 2 for the $m_{b l} e^{\operatorname{minimax}}$ variable defined as the minimum of the two maximum values of the invariant masses of the leptons and b-jets combinations. State-of-the-art predictions that naturally incorporate interference effects provide the best description of the data in the measured region of phase space most sensitive to these effects. These results provide an important constraint on interference models and will guide future model development and tuning.
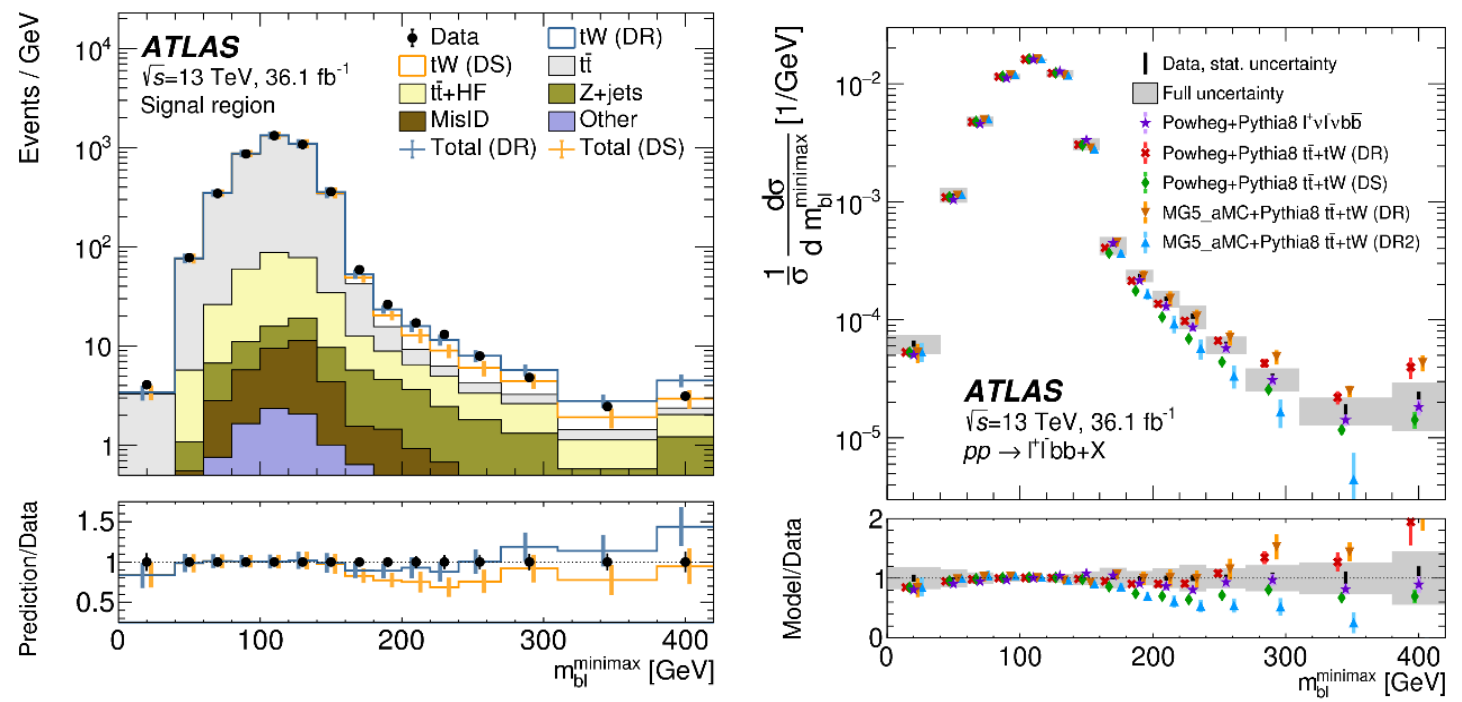

Figure 2: Left: The detector-level $m_{b \ell^{\text {minimax }}}$ distribution. The total predicted events are shown for both the diagram removal $(D R)$ and diagram subtruction $(D S)$ definitions of the $t W$ process, with uncertainties on the respective estimates indicated by separate error bars. Uncertainties include all statistical and systematic sources. Right: The unfolded normalized differential $m_{b e}{ }^{\text {minimax }}$ cross section compared with theoretical models of the $t \bar{t}+t W b$ signal with various implementations of interference effects [11].

\section{Observation of tZq at $13 \mathrm{TeV}$}

Single-top-quark production in association with a $Z$ boson is measured by the ATLAS experiment at $13 \mathrm{TeV}$ using an integrated luminosity of $139 \mathrm{fb}^{-1}$ [12]. The measurement is done in the trilepton channel with events containing three isolated charged leptons and two or three jets, one of which is $b$-tagged. The main backgrounds are from ttz $Z$ and diboson production. Neural networks are used to improve the background rejection and extract the signal. The measured cross section, including non-resonant dilepton pairs with $m_{\ell+\ell}>30 \mathrm{GeV}$, is $97 \pm 13$ (stat) \pm 7 (syst) fb, consistent with the SM prediction. Other measurements of single-top-quark production in association with a boson are carried out by the CMS Collaboration $[13,14]$.

\section{References}

[1] ATLAS Collaboration, JINST 3 (2008) S08003.

[2] CMS Collaboration, JINST 3 (2008) S08004.

[3] ATLAS and CMS Collaborations, JHEP 05 (2019) 088.

[4] CMS Collaboration, JHEP 10 (2018) 117.

[5] ATLAS Collaboration, JHEP 01 (2018) 63.

[6] CMS Collaboration, CMS-PAS-TOP-19-003, http://cds.cern.ch/record/2712818

[7] ATLAS Collaboration, EPJC 78 (2018) 186.

[8] CMS Collaboration, PLB 800 (2019) 135042.

[9] ATLAS Collaboration, JHEP 04 (2017) 086.

[10] CMS Collaboration, EPJC 80 (2020) 370.

[11] ATLAS Collaboration, PRL 121 (2018) 152002.

[12] ATLAS Collaboration, JHEP 07 (2020) 124. 
[13] CMS Collaboration, PRL 122 (2019) 132003.

[14] CMS Collaboration, PRL 121 (2018) 221802. 